\title{
Spatial Regression of reproduction of dengue fever mosquito's transmitter phenomenon in the university municipality-Jeddah province - Kingdom of Saudi Ariba -2018
}

\author{
Farah Abdallah Mohammed Elhassan ${ }^{1}$, Mubarak H.Elhafian ${ }^{12}$, Mohammedelameen Eissa Qurashi ${ }^{1 *}$ \\ ${ }^{1}$ Sudan University of Science \& Technology, Faculty of Science, Department of Statistics \\ ${ }^{2}$ King Abdul-Aziz University, Collage of Science and Arts Department of Mathematics
}

\begin{abstract}
The method of Geographical Information System is used for building statistical models to estimate the number s of mosquitoes which are effected by natural factors. Decisions makers and mosquitoes' combater; in particular, those carriers to Dengue Fever, regarding to set plans and methods to fight them depending on the weighted least square model.

The study aimed to build a model known by regressions spatial to the phenomenon of mosquitoes' reproduction within the neighborhood of the Jeddah Municipal. Also, studying the quality of spatial models which is estimated; including studying and determining reasons that lead to mosquitoes' reproduction carry Dengue Fever within Jeddah City. The researcher also did not forget the possibility of calculating or estimating the numbers of mosquitoes carry Dengue Fever in any single spot within the neighborhood whereas two factors were determined which are the temperature and humidity to estimate numbers of mosquitoes carry Dengue Fever. Data were collected to average of the numbers multiplied mosquitoes carry Dengue Fever in Jeddah City in weeks from (1-45) year 2018 including the average of temperature and humidity for the same period; whereas the determined Coefficient was $\mathrm{R}^{2}=55 \%$ and the result of that model showed fifty five percent of the total changes happen in mosquitoes reproduction within the municipal of Jeddah K.S.A. AIC method was used to select the best model. Whereas the residuals in the model showed to un-annotator model and they were determined that return to the other environmental affect which are considered to be the foci to mosquitoes reproduction because of the way of water overstocking, sanitation, swamp of wasted water and residents' customs and traditions; whereas the targeted study area has various numbers of foreigners from different parts of the world which made a differences and variety in customs and traditions.
\end{abstract}

Key words: Geographical weighted Regression, Bandwidth Method, Cross Validation, Dengue Fever, Moran's Index

\section{Introduction}

We see many and different phenomenon in our everyday life effect by some factors regarding to increasing or decreasing, which cause statistical relationships which need suitable statistical method or methods to suit the type of data; whereas we find that the regression is one of these statistical methods which determined the form and direction of the relationship (Seber,Wild .2003).; so it used widespread in many economical, sociological, environmental fields (Rawlings, et al 2001). and it answers the relationship question between two variables or more; is this relationship positive one or negative? What are the most important elements or 
variables that effect in finding an arithmetical relationship between a groups of variables? What are the Influential leads to specific result? Where does this relationship increase and where does it decrease? One of the two variables is called dependent while the other one is independent which is the cause in changing the dependant, in such case it calls ( Simple linear Regression) which can be more than one variable, in this case it calls ( Multiple linear Regression) (Montgomery et al.2012). Furthermore the two types of regressions one of the advanced statistical method that insure accuracy in adherent for improving the research results through the optimal usage of data in finding casual relationship between the phenomena of this subject of the study.

Because of the change that occurred on the environment and the increase in population which effect negatively or positively on the natural environmental phenomenon within the extend geographical area whereas a need to show the effect of the spot phenomena protrudes on the increase or decrease of the phenomena on a specific geographical spot; and to what extend its difference on the other spots. This kind of regression is called multi spatial regression which is an attempt to find an equalization to the regression which enters dimensional spatial ( spot's abscissas ) within it, it cares of element value or that appeared in a specific geographical point or the spatial spot $(\mathrm{x}, \mathrm{y})$ whereas it finds the equalization of the regression appliances to apparent spots.( Mauricio Bini et al 2009).

\section{The Problem of the study:}

Statistical phenomenon usually is affected by the environmental factors such as rains, temperatures, humidity and other factors, but these factors are vary from an area to another which affect the phenomenon ( might refers to behavior attitude and people's tradition in every geographical area whether a neighboring one or not; including administrative and political procedures regarding taking care of some places of interests in fighting some phenomena, increasing people's a awareness and to what extend are health services essentials available ) all those reasons lead to existence of complicated relationship when gathering samples that ignore the spatial dimension (the spot) for each single sample there for we need statistical methods that include the spatial in the statistical process .

The phenomena of mosquitoes reproductions and multiplication, in particular that carrier to Dengue Fever, within the neighborhood of Jeddah Municipal could be moving between neighboring neighborhood because of the spatial neighboring with the abundance of all fighting methods within any single neighborhood. We find that each municipal has its own many subordinate factors regarding mosquitoes multiplication such as environmental factors and population demographic; whereas the so we use statistical methods that include the spot in the statistical operations came out which participate in estimating the numbers of mosquitoes that carry Dengue Fever in every single spot within the Municipal of the Campus's neighborhood.

\section{Theoretical Framework:}

\subsection{Spatial Regression:}

The spatial Regression Analysis is trying to find an answer to the relationship between tow variables or more, whereas this relationship a positive one or negative? The elements or variables that effect the mathematical relationship between the spatial variables and the factors or influential lead to a specific result. Spots variables increase and decrease.( Tiefelsdorf, 2006).

It is a statistical method searches how to find regression equalization which enters the spatial dimension the axis $(\mathrm{x}, \mathrm{y})$ and we have a specific element changes within spatial spots with weighted abscissas and these values in these points represent the samples.

There are various types of the Spatial Regression Analysis concern of the relationship between a variable or more, whether these variables are positive (rejected) or negative ( contrastive ). The most important 
elements or variables that effect finding the Mathematical Relationship between two groups of spatial variables also the effective factors lead to a specific result such as increasing or decreasing within a specific places. It is possible to compare multi spatial Regression to the common multi linearity regression as follow:

- The Common Multi Linear Regression is an equation for each study area, however the Spatial Regression is one changing equation to each part (a spot) from the study area ( the value of variables are spatially changed).

- The Common Multi linear Regression its variable values are stable, while the data of Spatial Regression changes according to the spot.

\subsection{Geographical Weighted Regression:}

The Geographical Regression is considered to be one of the local regression models ( or the favorite ) most used one, whereas it is connected to one variable depends on independent variables groups and it is considered to be one of the most important regression's egression because it estimated as a marked one that connect every single independent variable on accredited one.( Brunsdon, et al .1998).

The Geographical Weighted Regression is the advanced statistical technology that allows the operations sampled spatially. In other words, GWR implement the regression to each single site replacing the only regression for the whole study area, whereas there will be a spatial distribution to the GWR's results. There are three reasons at least we think that the relationship between the variables will vary from first to last place.(( Fotheringham, et al .2003).

Firstly: there is no doubt there is spatial difference to the samples because of the random variables samples that participate to this source which will be unconventional spatially, whereas these differences do not have great effect by itself but it should be taken in consideration.

Secondly: human attitude and behavior and residents" practicing including their attitudes and favors, add to that their political and administrative issues, whereas these behaviors and residents' practice differ from place to another, and the result of that the necessity of taking care of the spatial dimension on the contrary to quantum analysis which ignore concerning spatial dimension without involving the spatial complications. The spatial treatment method relating to the spot is treated by respond to these complications and critics which means responding to these critics.

Thirdly: Misconception to the model because of data gathering does not represent the reality usually; and any deletion leads to misrepresentation, that is why GWR is considered to be a method aids researchers and decision makers in distinguishing the local characteristics effects. Through using local model (the partial ) and the earth querying model are not classical can easily obtain through which accurate local indications.

Weighted linear Regression analysis is used normally in sociological and economical studies also it uses in geographical and health studies.

Figure (1) geographical regression tests spatial treatments to every independent variable in a form of a map
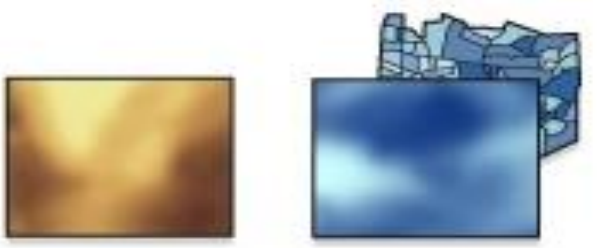

$\mathrm{B}_{1}$

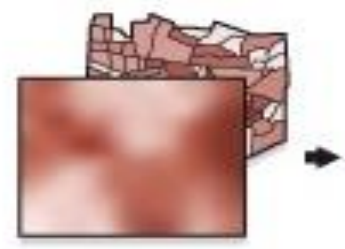

B?

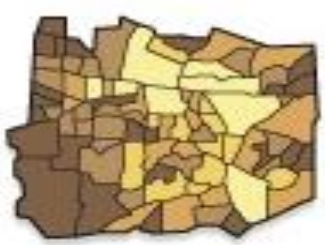

v 
According to the equation:

$$
y_{i}=B_{0}+\sum_{i=1}^{n} B_{K} x_{k i}+\varepsilon_{i}
$$

Where yi is the independent variable, Bk the coefficients, Xki the independent variables, and $\varepsilon \mathrm{i}$ the error term.

GWR enable local variations (over space) in the estimation of coefficients, thus the regression coefficient Bk takes different values for each location. This method generates a separate regression equation for each observation, which can be expressed as follows:

Where yi is the dependent variables, Bk the coefficients Xki the independent variables; ( ui , vi ) co ordinate location of $\mathrm{i}$, and $\varepsilon \mathrm{i}$ the error term.

The estimator of term takes the form of:

$$
\dot{B}=\left(X^{T} W\left(u_{i}, v_{i}\right) X\right)^{-1}\left(X^{T} W\left(u_{i}, v_{i}\right) Y\right)
$$

Where W (ui, vi) is square matrix of weights relative to the position of ( ui, vi ) in the study area. XT W ( ui , vi ) is the geographically weighted variance - covariance matrix ( estimation requires its inverse to be obtained) and $\mathrm{Y}$ is the vector of the values of the Coefficients Standards Error.

The matrix W (ui, vi) matrix contains the geographical weights in its leading and diagonal and (0) in it's offdiagonal elements.

$$
W(u i, v i)=\left(\begin{array}{cc}
W 1(u 1, v 1) & 0 \\
\cdot & \cdot \\
0 & W i(u i, v i)
\end{array}\right)
$$

Each equation is calibrated using the different weights of the observations contained in the data set. The assumption is that observations one near an anther have greater influence on each other's parameter estimates than observations farther apart, according to Table's law. The weight assigned to each observation is based on a distance decay function centered on observation i. (2) (Bao, et al. 2018)

Table (1):The difference between total statistical operations and the local statistical ones illustrated on below table (1)

\begin{tabular}{|l|l|}
\hline Total statistic & Local statistic \\
\hline Data are taken equally for the whole area & $\begin{array}{l}\text { Search within spatial differences to total } \\
\text { statistics }\end{array}$ \\
\hline One value Statistic & Multi values statistic \\
\hline Can't be represented by maps & Can be represented by maps \\
\hline It is not a ally to GIS & It is a ally to GIS \\
\hline Does not deal with place & Deals with place \\
\hline
\end{tabular}




\begin{tabular}{|l|l|}
\hline excessive to resemblances to place & Excessive to difference on place \\
\hline $\begin{array}{l}\text { Searches symmetric phenomena to extract } \\
\text { stabled equations }\end{array}$ & $\begin{array}{l}\text { Searches local exceptions to extract what } \\
\text { called hot spots }\end{array}$ \\
\hline
\end{tabular}

GWR's Interpretation:

The GWR's table includes a group of important factors which consists of:

a. Condition Numbers:

It is an standard for existence to what extend multi overlapping is between independence variables and the model's aptitude and quality; whereas if it is exceed 30th condition this implies that there is multi overlapping between independent variables which indicates incompetency and lack of quality for the model

$\mathrm{R}$-square $\left(\mathrm{R}^{2}\right)$ which determined the model's competency in interpreting the change within independence variables whereas its values vary between ( 0 to 1$)$ when it become zero that reveals the weakness of the model performance and vice versa if it distancing from zero to one shows the model strength regarding interpreting the phenomena. The independence variables have an active effect in creating an effect on the dependent variable .

Predicted Values:

It is the calculated values to the dependent variable which resulted from the regression.

Residuals:-

The difference between the observed original value and the predicted to the Coefficients Standards Error whereas whenever residuals' value become tiny that showed the strength and quality of the model.

Coefficients Standards Error:

Values for measuring the quality of every single estimation to the regression regarding every single area whether separated or a geographical patch whereas these values are tiny the regression will be best.

The kind of the kernel carry out the regression operation, by using: adaptive value as an extend and calculated through determining the number of required neighbor.

Bandwidth Method: it determines the extend of the kernel carry out the regression operation so as to find the best suitable distance or the suitable one to the numbers of the neighbors. The user here has to choose one method out of the two for searching or sample process (Guo, \& Zhang, 2008).

\section{Result and Discussion}

\subsection{Data Set:}

The data of the average of Mosquitoes' reproduction and multiplication were gathered within the university neighborhood in ( $1-45)$ weeks of the year 2018; including the humidity and temperature within the same period of this study. The laborers and some employees working for Asma Company for Environmental Solution - which specialized in Environment Studies and Mosquitoes' Fight - carried out this part of the study regarding the type that carry Dengue Fever in Jeddah City.

Figure (2) 


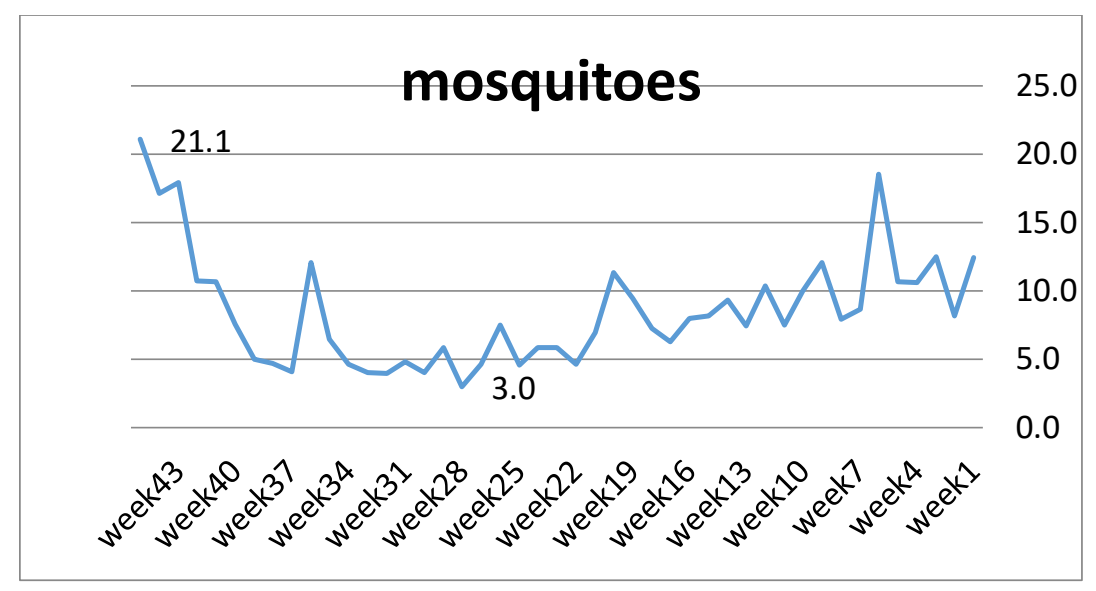

We can notice from Figure (2) that the spatial average for circulating the residuals vary between ( 21.1) in week forty fifth and (3) residuals in the week twenty eighth.

The reports that adjourning to those in charge were restricted within Jeddah Secretariat on some statistical descriptive and showing the numbers only and the concentration places of reproductions. The researcher tends to use some advanced spatial statistical methods and knowing the effect of the spot regarding mosquitoes' breeding including to some other spatial methods, whereas the data come as follows:

- The spatial average to the mosquitoes in the residuals within weeks $(1-45)$ through the year as Coefficients Standard Error,.

- $\quad$ The average to every week abscissas from week ( 1- 45 ) all the year long.

- The average of humidity for the weeks $(1-45)$ all the yearlong 2018 was independent variable

- Average of temperature for the weeks ( 1-45) all yearlong 2018 was independent variable

Which is spread as follows:

Table (2):Descriptive Statistic to the Variables:

\begin{tabular}{|l|l|l|l|l|}
\hline The variable & Greatest value & Less value & Average & $\begin{array}{l}\text { Aberrance } \\
\text { Apparatus }\end{array}$ \\
\hline $\begin{array}{l}\text { Number of } \\
\text { Mosquitoes }\end{array}$ & 21,078 & 2,95 & 8,53 & 4,12 \\
\hline Temperature & 30,094 & 23,3 & 36,1 & 3,65 \\
\hline Humidity & 68,7 & 34,2 & 51,43 & 8,01 \\
\hline
\end{tabular}

Source: Researcher Using : ArcGIS Number of sample 45

\subsection{Results:}

WGR was used to estimate the phenomena of mosquitoes carry Dengue Fever reproductions within the university neighborhood accrediting the two variables ( humidity and temperature ) as independent variables according to following procedures:

After following the special procedures regarding Geographical Data on Arc Map the Weighted Geographical Regression was calculated for each point of the mosquitoes' reproduction spots( each separated equation to any single spot) does the result of that a number of 45 separated equations on a table consists of : 
Observed Values to independent Variable which is the real value to the average numbers of the mosquitoes carry Dengue Fever within the neighborhood of the university through the weeks $(1-45)$ the year 2018.

Weights:

Weights were calculated to every single geographic spot whereas least value was less than( 2.954545) in the point (28) and the highest weight was on the spot (45) where the value reach (21,078292) distributed geographically as follows:

Figure (3)

\section{University Municipality Disticts}
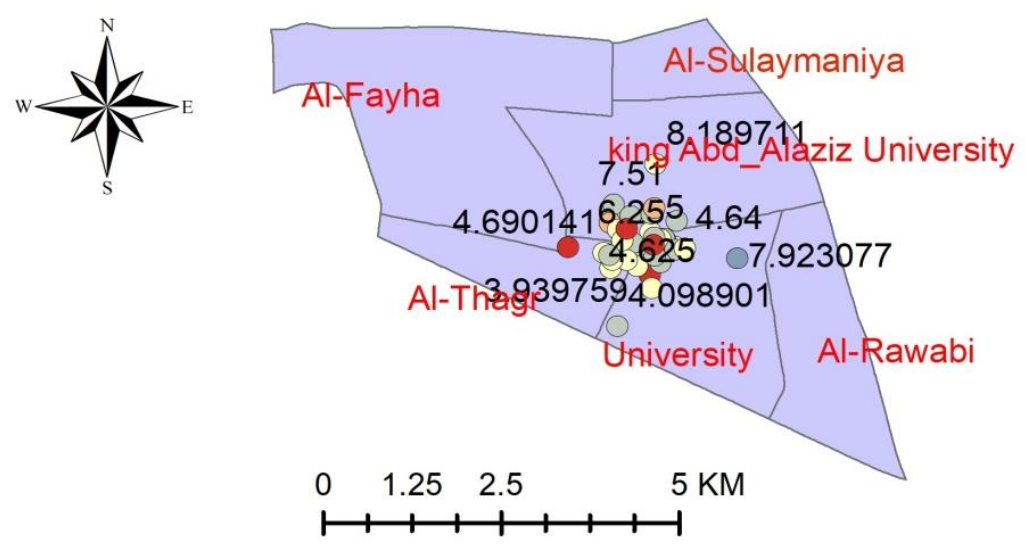

Conditions Numbers:

Which is a criterion to what extend is the linearity overlap to the independent variables; whereas we find that the condition numbers vary between ( $29 \mathrm{~m} 983$ to 18,028$)$ less than (30) does the result of that means nothingness of linearity overlapping between the independent variables. Geographically distributed within Jeddah Municipal Neighborhood as follows:

Figure (4)

\section{University Municipality Disticts}
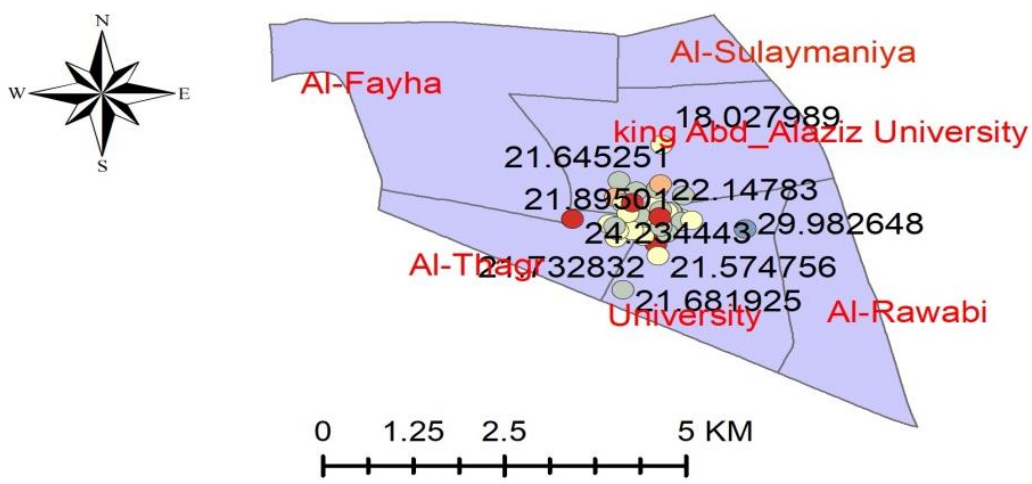

\section{R-Square:}

We find that the value vary between ( $0 \mathrm{~m} 237416)$ at the point $(8)$ as a least value and that means the average of humidity and temperature affect on mosquitoes carry Dengue Fever within the neighborhood reached 
(24\%) which is weak ratio. However the highest value to it reached $(0,610955)$ at point $(45)$ which means that the a proportion of temperature and humidity affect on mosquitoes carry Dengue Fever reproductions( the residuals ) at this point reached (64\%) which is a high affect at this point. It geographically distributed as follows:-

Figure (5)

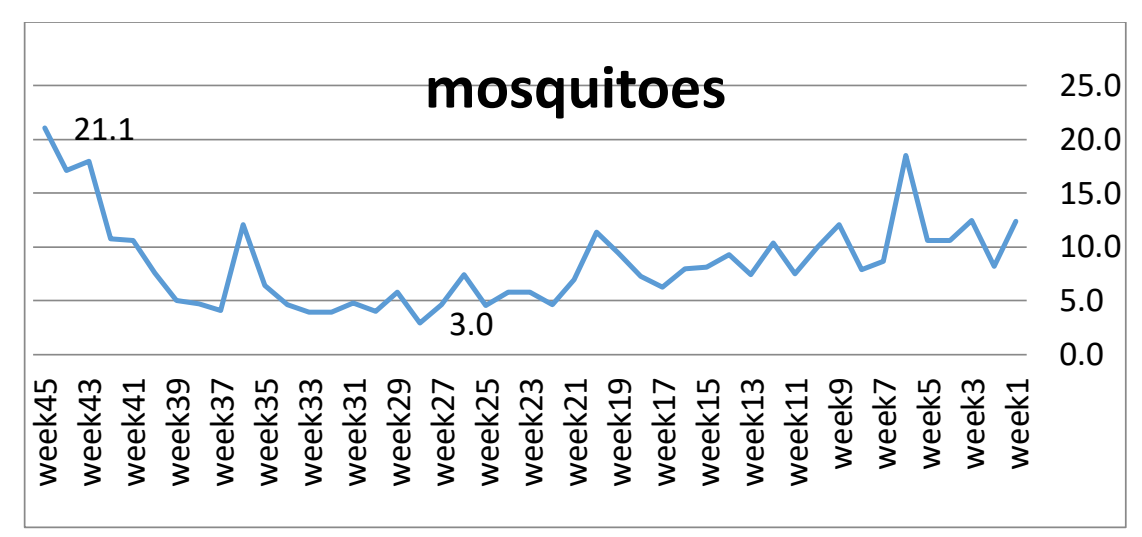

Figure (6)

\section{University Municipality Disticts}
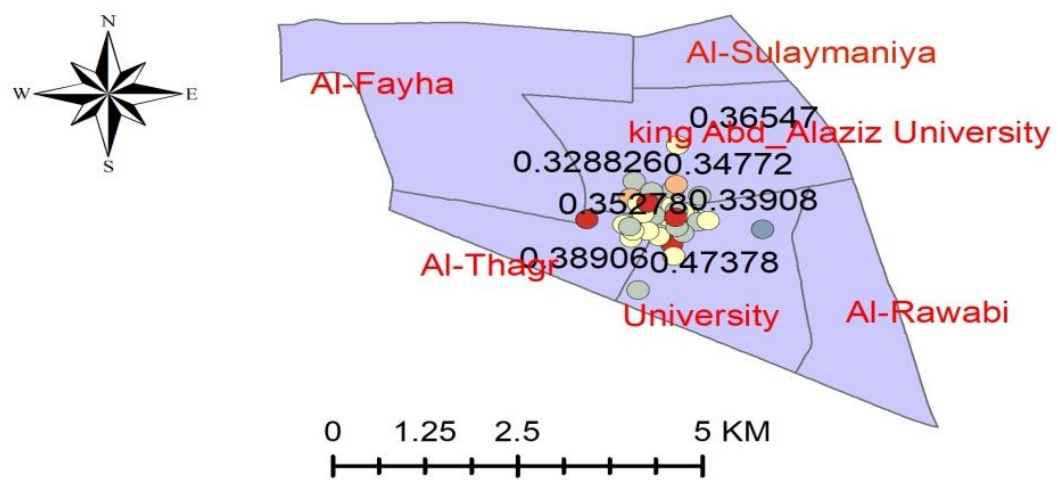

\section{Predicted Value: -}

It is the predicted value to the numbers of mosquitoes carry Dengue Fever ( the Residuals ) which is resulted out of the regression equation for each single one, whereas we find that the highest value reached $(17,758)$ at the point (45) in contrast we find least value reached (4.3.5) at the point (30). Geographically distributed as follows:

Figure (7)

\section{University Municipality Disticts}
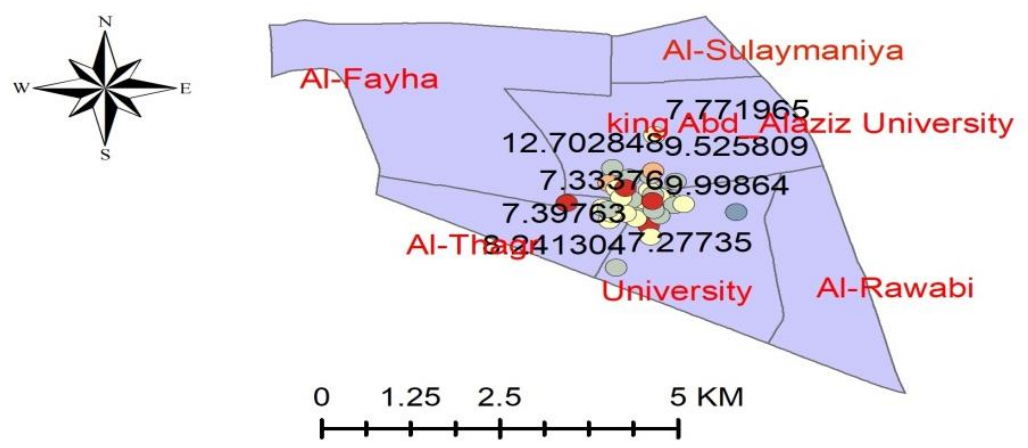


\section{Coefficients:}

a. Intercept Coefficient:

the value vary from ( 6.148) and (23.015) which is an average to the numbers of mosquitoes when taken in consideration the humidity and temperature, each of independent variables equal zero . distributed geographically as follows:

Figure (8)

\section{University Municipality Disticts}
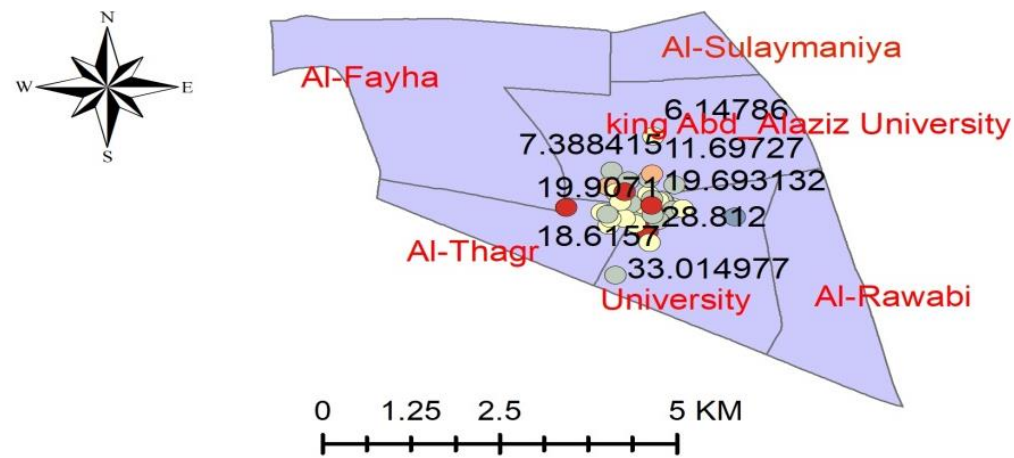

b. Temperature Coefficient:-

Its values vary from $(0,98-0,39)$ which means that the increase in temperature as a degree leads to decreasing in reproduction of mosquitoes carry Dengue Fever in an average of $(0,98)$ as a least and as a highest a quantum decreases $(0,39)$ as an highest quantum. Geographically distributed as follows within the neighborhood of the university municipal:

Figure (9)

\section{University Municipality Disticts}
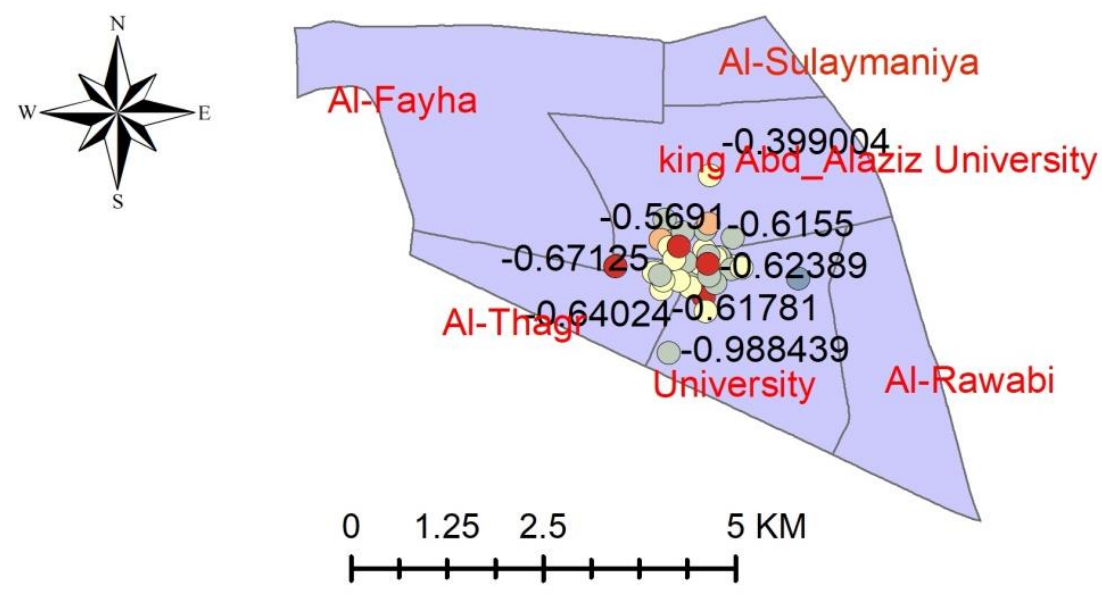

c. Humidity Coefficient:-

Vary between $(0,080,461)$ as least and $(0,386798)$ as a highest value to humidity. That means the increase in humidity as on degree leads to decrease in reproduction of mosquitoes carry Dengue Fever $(0,0805)$ at the point (8) whereas increases in average regarding humidity a degree leads to increase in mosquitoes carry 
Dengue Fever multiplication( the adult) within Jeddah municipal by $(0,3868)$ at the point $(45)$ geographically distributed as follows:

Figure (2)

\section{University Municipality Disticts}
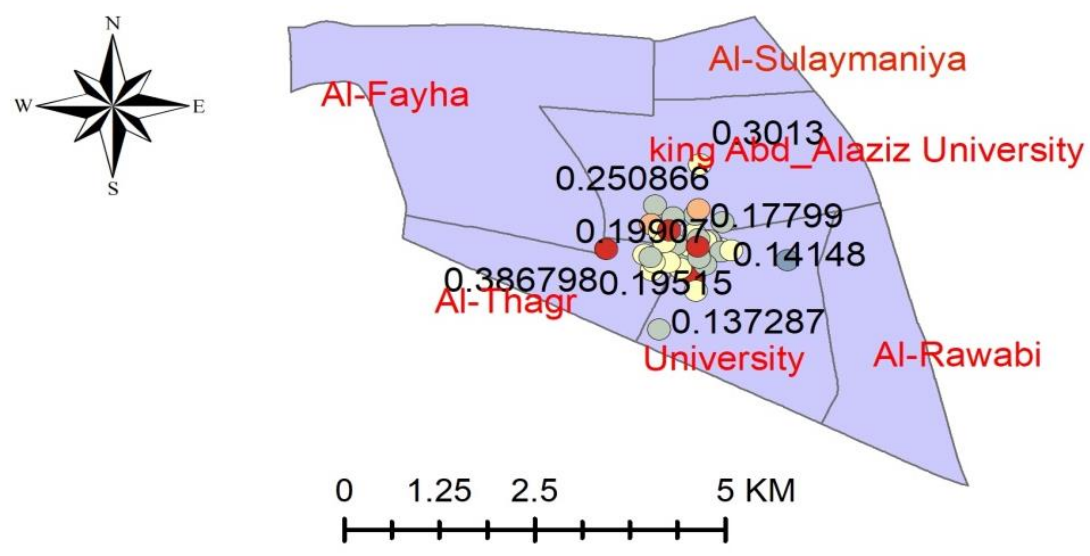

\section{Residuals:-}

It is the different between the real seen values and the expected output from the predicted operation , whereas we find that the highest value to the difference reached $(7,61)$ at the point $(43)$ and the least value to the difference reached $(-0,01)$ at the point $(2) \ldots$ geographically it is distributed as follows:

Figure (11)

\section{University Municipality Disticts}
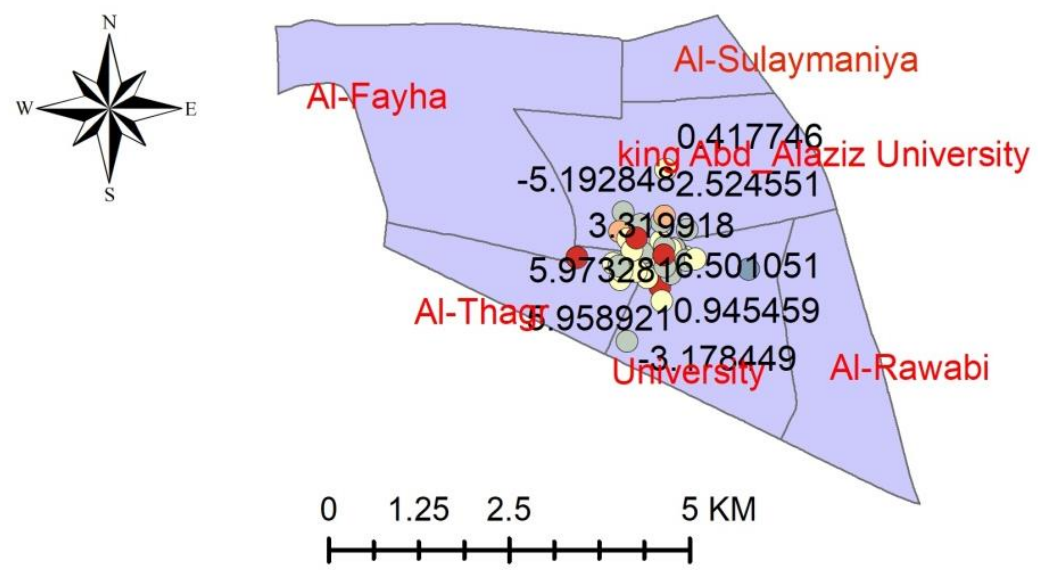

\section{Standard Error to the Sample:-}

We find that the least value reached $(1,43)$ and this is an abdication that the regression at point $(8)$ is considered to be of the best equation because of the quality of the sample, while the greatest value to the error standard reached $(2,95)$ at the point (12) which means that it was the weakest sample to the reproduction phenomena to the mosquitoes carry Dengue Fever (larva period ) within the university municipal, geographically distributed as follows: 
Figure (12)

\section{University Municipality Disticts}
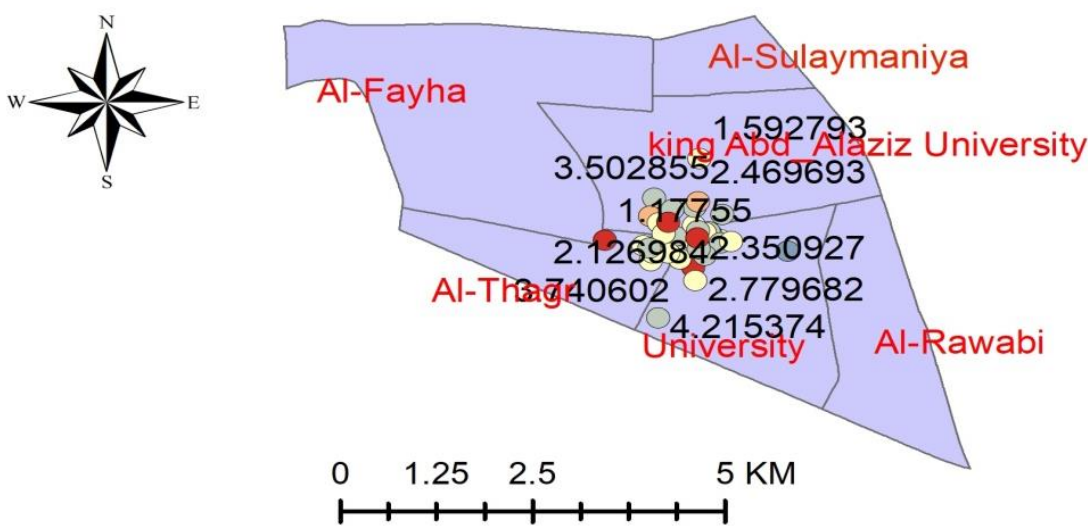

\section{Standard Error Intercept:}

We find that the values vary between (7.27) at the point No ( 7 ) as the biggest value, while the least value reached $(5,01)$ at the point No (28).

We observe that the values are somehow raising, geographically distributed as follows:-

Figure (13)

\section{University Municipality Disticts}
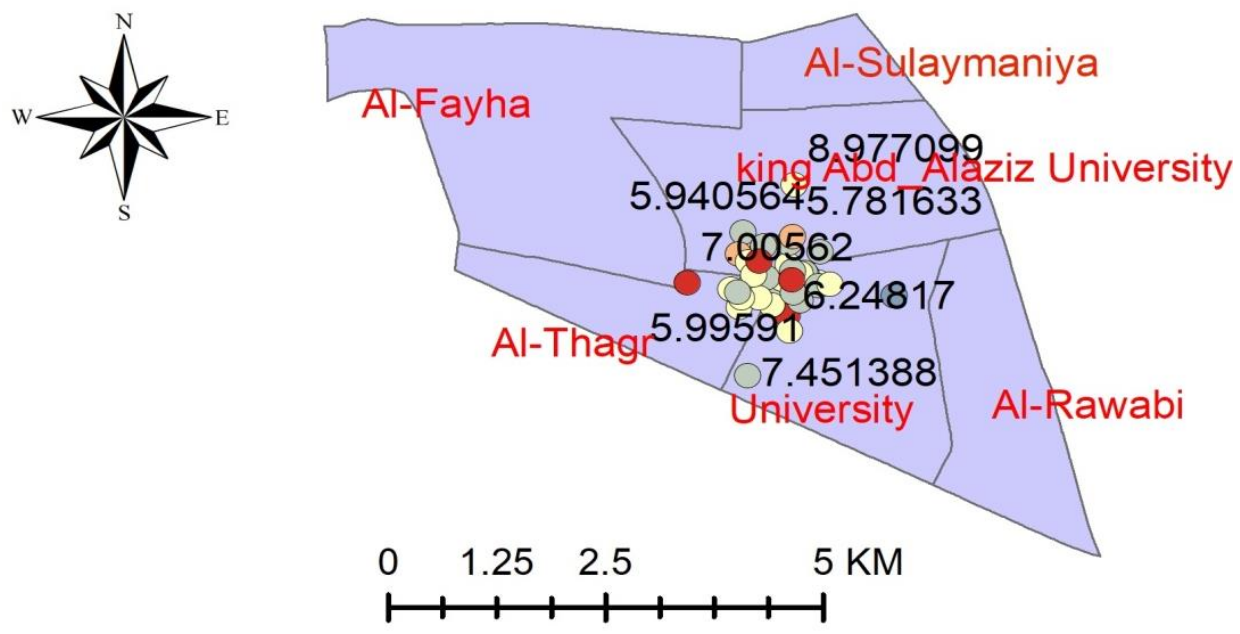


\section{Temperature Standard Error: -}

The Intercept Normative to Temperature reached $(0,13030)$ at the point $(16)$ as least value while the greatest value reached $(0,21634)$ at point $(8)$, we observe that the values decrease and geographically distributed as follows:

Figure (14)

\section{University Municipality Disticts}
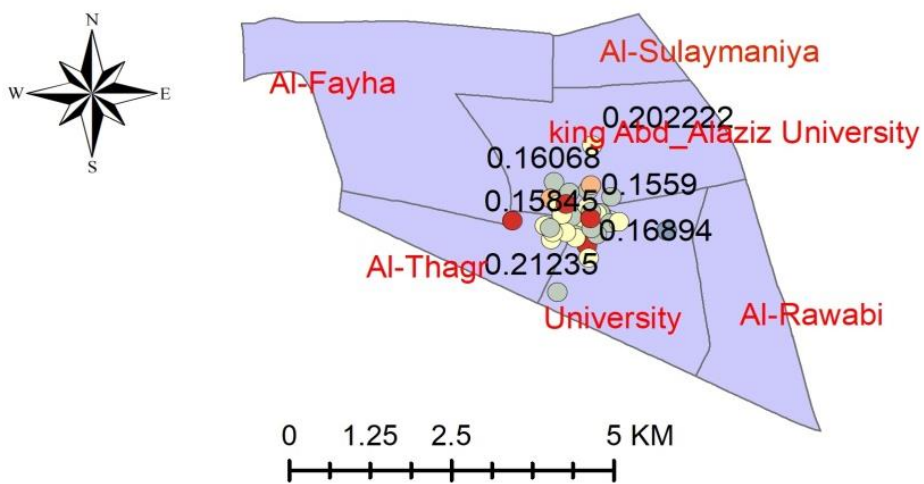

\section{Standard Error Humidity: -}

The Intercept Normative to humidity $(0,05959)$ at point $(43)$ as least value, whereas the greatest value reached $(0,08789$ ( at point $(7)$, we observed that the values reduced, and geographically distributed as follows:

Figure (15)

\section{University Municipality Disticts}
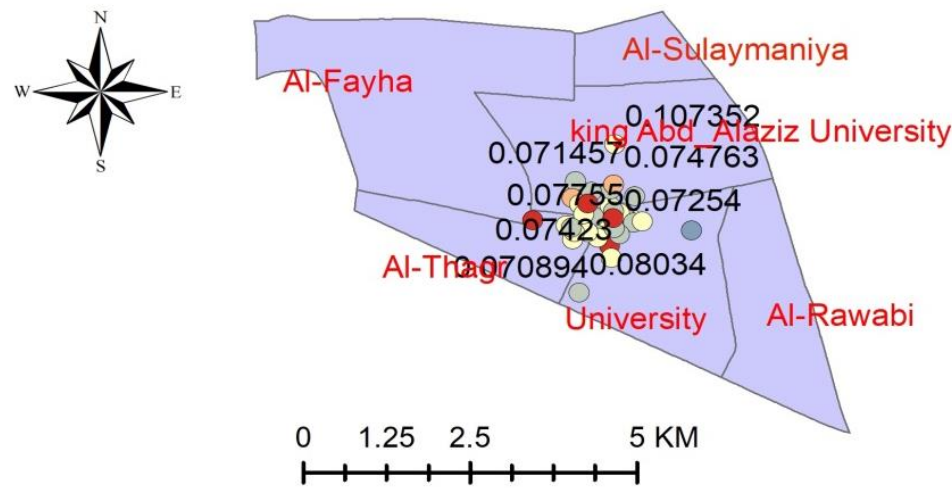

Standard Residuals values: 
The least value to the residuals reached $(-0,008)$ at point $(2)$. However the highest value reached $(2,587)$ as biggest value to the residuals at point ( 44 ) which is least values distributed as follows:

Figure (16)

\section{University Municipality Disticts}
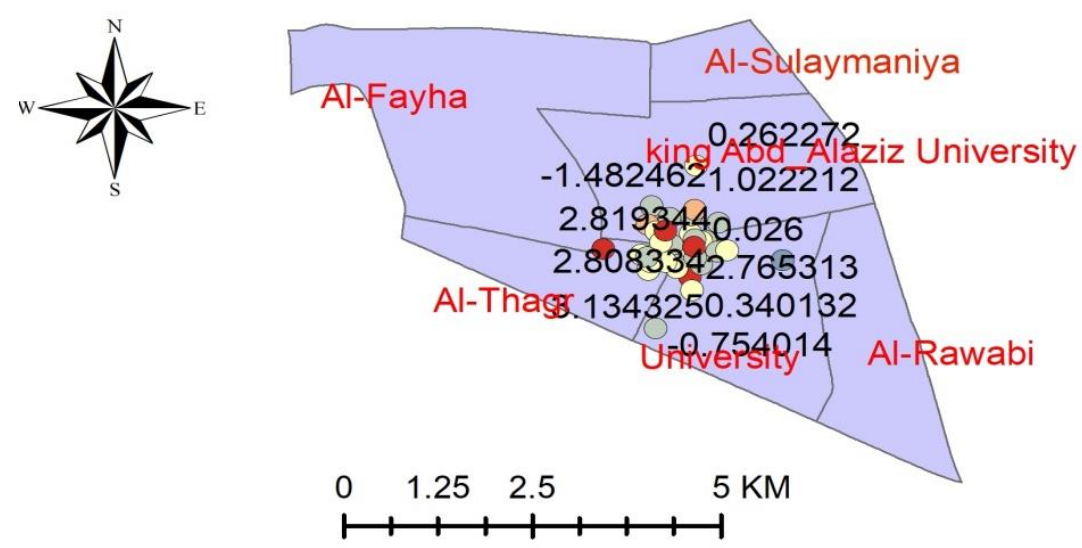

\section{Bandwidth}

Bandwidth or neighbor number: it is the value the program calculated it for each modulating operation for the samples regression for the best sample where we find it totaled $(0,008087)$.

\section{Residuals Squares:}

The value of total remaining residuals squares in the sample reached $(342,474,32)$

\section{Effective Number:}

Reflects the bargains between the discrepancy in the sample values and the value of aberrance regarding moduli estimation whereas it reached $(7,43574)$.

Sigma Value: Value of the standard quadratic root for the major remaining or the value of the normative aberrance to the remaining and they reached (3,01944)

AICs Modulus reached ( 235,405399)

$\mathrm{R}^{2}$ Sample Modulus Competency ( 0,551687)

Formative corrected Modulus ( the adjusted ) reached ( 0,474879)

To attain to what extend the random errors mean something, a statistical modulus is used ( Moran's Index) knowing to what extend is the concentration of the residuals.

Table (3):Random of Errors Distribution Test:

\begin{tabular}{|l|l|}
\hline Moran's Index & 1,000 \\
\hline \hline Z-score & 70,9174 \\
\hline P-value & 0,0000 \\
\hline
\end{tabular}

Figure (17) 


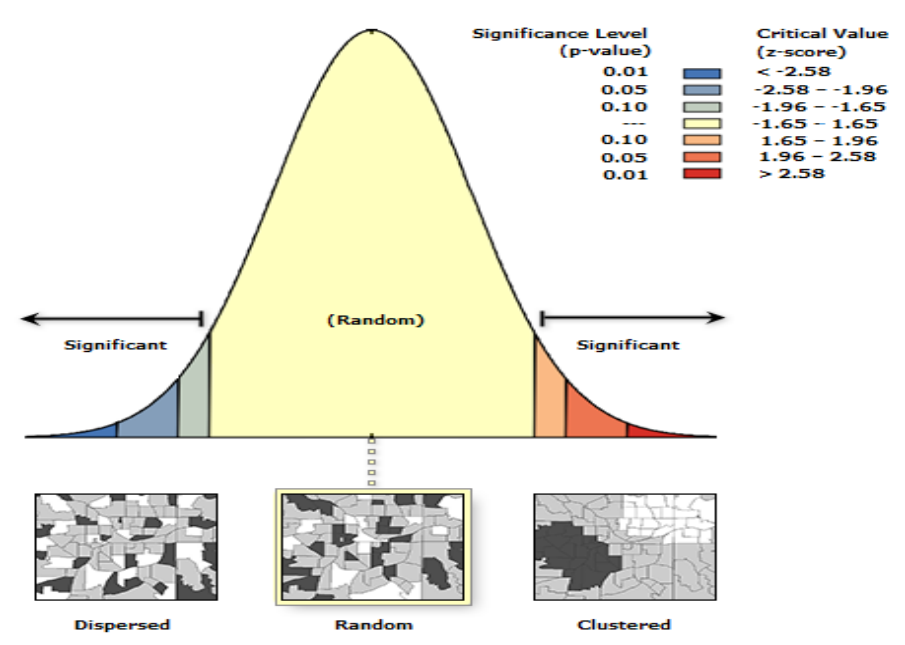

The Test of the Quality of the Spatial Models:

To know the intangibleness of the conduct, the calculation $(t)$ has been run to each modulus of the sample ( B0 , B1 , B2 ) according to the following equation:

The results was as the same as the attachments:

From table $(\mathrm{t})$ the value of table has found statistically $(0,05)$ and a degree $(44)$ whereas we find it reached $(1,671)$ we find the transaction's values $(\mathrm{t})$ calculated to the fixed rim $(\mathrm{B}())$ maximum than the table one whereas it varies between $(16,82)$ at the point $(8)$ as a maximum value and $(4,84)$ as a minimum at the point (11) which means that the fixed rim is incorporeally at all points.

That why the comparison of temperature moduli at all points find that the value scheduled to $(\mathrm{t})$ greater than the accounted value to the (B1) temperature at all points which means that there is no relationship of statistical denote to the temperature regarding mosquitoes reproductions.

When comparing the humidity ratios at all points we find that the scheduled value to $(\mathrm{t})$ is greater than calculated value for (B2) humidity at all points and this means that there is no statistical relationship to the humidity behind mosquito's multiplication.

Compare between spatial models:

We will compare between three spatial models as table below:

Table (4):

\begin{tabular}{|l|c|c|c|c|}
\hline Location & B & S.E & T & \multirow{2}{*}{ R-square } \\
\hline \multirow{4}{*}{ King Abd-Alaziz University } & 6.1479 & 8.977 & 0.684843 & \\
\cline { 2 - 4 } & -0.399 & 0.202 & -1.97329 & \multirow{3}{*}{0.36} \\
\cline { 2 - 4 } & 0.3013 & 0.107 & 2.8054 & \\
\cline { 2 - 4 } University district & 22.87 & 5.915 & 3.866441 & \multirow{2}{*}{0.3411} \\
\cline { 2 - 4 } & -0.6698 & 0.165 & -4.05939 & \\
\cline { 2 - 4 } & 0.1415 & 0.077 & 1.83053 & \\
\cline { 2 - 4 } Al-Thagar & 11.891 & 7.006 & 1.697259 & \multirow{2}{*}{0.611} \\
\hline
\end{tabular}

From table above we can built model for each three locations as: 


$$
\begin{gathered}
\hat{y}=B_{0}+B_{1} x_{1}+B_{2} x_{2} \\
\hat{y}=\text { number of mosqutios } \\
x_{1}=\text { Averagetemperatures } \\
x_{2}=\text { Average humidity }
\end{gathered}
$$

1. King Abd-Alaziz University:

$$
\hat{y}=6.1479-0.399 x_{1}+0.313 x_{2}
$$

6.1479: is the averages number of mosquitoes, when (humidity and temperatures) zero or fixed, at King Abd-Al-aziz University area.

-.399: the increasing in temperatures one unit, leads to decrease in mosquitos breading with (39.9\%), at King Abd-Al-aziz University area.

0.313: the increasing in humidity one unit, leads to increase in mosquitos breading with (31.3\%), at King Abd-Al-aziz University area.

2. University District:

$$
\hat{y}=22.87-0.6698 x_{1}+0.1415 x_{2}
$$

22.87: is the averages number of mosquitoes, when (humidity and temperatures) zero or fixed, at University district.

-.6698: the increasing in temperatures one unit, leads to decrease in mosquitos breading with (66.98\%), at University district.

0.141 : the increasing in humidity one unit, leads to increase in mosquitos breading with (14.15\%), at University district.

3. Al-thagar:

$$
\hat{y}=11.891-0.693 x_{1}+.3863 x_{2}
$$

11.891: is the averages number of mosquitoes, when (humidity and temperatures) zero or fixed, at Al-thagar district.

-.693: the increasing in temperatures one unit, leads to decrease in mosquitos breading with (69.3\%), at Al-thagar district.

0.3863: the increasing in humidity one unit, leads to increase in mosquitos breading with (38.63\%), at Al-thagar district.

Table (5): shows these spatial models:

\begin{tabular}{|l|c|}
\hline Points (districts) & Spatial models \\
\hline $\begin{array}{l}\text { King Abd-Alaziz } \\
\text { University }\end{array}$ & $\hat{y}=6.1479-0.399 x_{1}+0.313 x_{2}$ \\
\hline University district & $\hat{y}=22.87-0.6698 x_{1}+0.1415 x_{2}$ \\
\hline Al-thagar & $\hat{y}=11.891-0.693 x_{1}+.3863 x_{2}$ \\
\hline
\end{tabular}

The table shows the relative importance for each model of three locations (Kin Abd-Alaziz University, University district and Al-thagr). It is seems clearly that King Abd-Alaziz University area there is an equivalent (equal integer one) between the degree of temperature and humidity on 
their impact on mosquito reproduction. In University district residential the temperature degree impact reached 5 times humidity degree and for Al-thagar district gets two time average .

There for we can conclude that from these models King Abd-Alaziz University area is the best among these areas because it doesn't dace any environmental problems (sanitation , informal housing ... etc ) that affect mosquito reproduction, then Al- thagar district area where the temperature and humidity degree are equivalent this is represent a weakness and that means availability of such environmental problems, but the university district area got the third level which means there is more environmental problems that face this district so we find that the relevant importance for independent variable reached five times .

\subsection{Discussion:}

-The GWR produced to us th estimation of mosquitoes numbers that carry Dengue Fever within the university Municipal in Jeddah City, total number of 45 samples by numeral average of Geographical Variable.

-Determining Moduli to the spatial model which is estimated vary between (35\%) to (57\%) that means the interpreted power to the independence variables humidity and temperature degrees between weak to medium one.

- The value of total determined modulus (55\%) that means the sample interpreted quantum of fifty five percent of the variable occurred in the number of the mosquitoes carry Dengue Fever within the university municipal.

-The results showed that temperature effects on mosquitoes reproductions was negative effect, on other words, increase in temperature leads to decrease in the numbers of mosquitoes that carry Dengue Fever within the university municipal.

-The result of the study showed that the humidity effect was positively whereas the increase in humidity leads to increase in the ratios of reproduction of the mosquitoes carry Dengue Fever within the university municipal.

-Inference could be said that through the output maps with modulus of calibration that the ratios of the effect of stable variables (humidity and temperature) increase whenever we tend west to the municipal and decrease whenever we tend east.

-The results showed that there is no incorporeally effect to the variables (humidity and temperature ratios ) in the phenomena of mosquitoes' breeding within the municipal.

-From the resulted map ratiocinate that by GWR that the spots need more fighting operation and that is for the reality of classification has to do with residuals; whenever these residuals are big this means that the spots need more studies and search to others environmental factors that assist in mosquitoes carry Dengue Fever reproduction, which are not included in the sample such as ( numbers of swamps, water sanitation, ways of storing water, water produced from Air conditioners and etc. ... which may return to the residents' customs and traditions within the university municipals.

\section{Conculation}

-Depending Geographical Informational Systems in putting a policy to fight mosquitoes carry Dengue Fever within the university municipal and the other municipals within Jeddah City in general. 
-The importance of fighting mosquitoes outcropped according the plans the municipal set. Workshops and awareness colloquium should be launched to Jeddah residents to reduce mosquitoes reproductions.

-The study discussed spots and areas of mosquitoes carry Dengue Fever reproduction within Jeddah Municipal whereas the study learns two factors could be interpreted which are humidity and temperature. The learners and researchers should study the other environmental factors of relation to mosquitoes that carry Dengue Fever reproduction in the entire municipal of Jeddah provinces and in particular the university municipal.

\section{Refrences:}

[1] Seber, G. A., \& Wild, C. J. (2003). Nonlinear Regression. Hoboken. New Jersey: John Wiley \& Sons, 62, 63.

[2] Rawlings, J. O., Pantula, S. G., \& Dickey, D. A. (2001). Applied regression analysis: a research tool. Springer Science \& Business Media.

[3] Montgomery, D. C., Peck, E. A., \& Vining, G. G. (2012). Introduction to linear regression analysis (Vol. 821). John Wiley \& Sons.

[4] Mauricio Bini, L., Diniz-Filho, J. A. F., Rangel, T. F., Akre, T. S., Albaladejo, R. G., Albuquerque, F. S., ... \& Isabel Bellocq, M. (2009). Coefficient shifts in geographical ecology: an empirical evaluation of spatial and non-spatial regression. Ecography, 32(2), 193-204.

[5] Tiefelsdorf, M. (2006). Modelling spatial processes: the identification and analysis of spatial relationships in regression residuals by means of Moran's I (Vol. 87). Springer.

[6] Brunsdon, C., Fotheringham, S., \& Charlton, M. (1998). Geographically weighted regression. Journal of the Royal Statistical Society: Series D (The Statistician), 47(3), 431-443.)

[7] Fotheringham, A. S., Brunsdon, C., \& Charlton, M. (2003). Geographically weighted regression: the analysis of spatially varying relationships. John Wiley \& Sons.

[8] Bao, J., Shi, X., \& Zhang, H. (2018). Spatial analysis of bikeshare ridership with smart card and POI data using geographically weighted regression method. IEEE Access, 6, 76049-76059.

[9] Guo, L., Ma, Z., \& Zhang, L. (2008). Comparison of bandwidth selection in application of geographically weighted regression: a case study. Canadian Journal of Forest Research, 38(9), 2526-2534.

[10] Guo, L., Ma, Z., \& Zhang, L. (2008). Comparison of bandwidth selection in application of geographically weighted regression: a case study. Canadian Journal of Forest Research, 38(9), 2526-2534.

[11] Páez, A. (2005, May). Local analysis of spatial relationships: A comparison of GWR and the expansion method. In International Conference on Computational Science and Its Applications (pp. 162-172). Springer, Berlin, Heidelberg.) 